\title{
Periodic Fetal Heart Rate Pattern
}

National Cancer Institute

\section{Source}

National Cancer Institute. Periodic Fetal Heart Rate Pattern. NCI Thesaurus. Code C111775.

Fetal heart rate patterns associated with maternal uterine contractions. 